\title{
Evaluation of High Resolution Computed Tomography Findings and its Relationship with the Clinical Course of Patients with Covid-19
}

Cyrus Emir-Alavi

Guilan University of Medical Sciences

Zakiyeh Jafaryparvar

Guilan University of Medical Sciences

Mohammad Amin Khajavi Gaskarei

Guilan University of Medical Sciences

Hossein Hemmati

Razi Hospital, Guilan University of Medical Sciences

Tofigh Yaghubi Kalurazi

Guilan University of Medical Sciences

Ali Alavi Foumani

Razi Hospital, Guilan University of Medical Sciences

Siamak Rimaz

Guilan University of Medical Sciences

Abbas Sedighi-Nejad

Guilan University of Medical Sciences

Hoda Sabati

Guilan University of Medical Sciences

Behnaz Ghanbar-Moghaddam

Guilan University of Medical Sciences

Hossein Khoshrang

Guilan University of Medical Sciences

Maryam Ahmadpour

Guilan University of Medical Sciences

Seyedeh Maral Mousavi

Guilan University of Medical Sciences

Hadi Sedigh Ebrahim-Saraie

Guilan University of Medical Sciences

Zahra Golamalipour Garfami

Guilan University of Medical Sciences

Zobin Souri ( $\sigma$ vahid.s13@gmail.com ) 
Guilan University of Medical Sciences

\section{Mohaya Farzin}

Guilan University of Medical Sciences

\section{Research Article}

Keywords: Covid 19, High-Resolution Computed Tomography, clinical

Posted Date: March 22nd, 2021

DOl: https://doi.org/10.21203/rs.3.rs-289858/v1

License: (c) (i) This work is licensed under a Creative Commons Attribution 4.0 International License. Read Full License 


\section{Abstract}

Background: Due to the Covid 19 pandemic and the lack of useful information, in this study, we evaluated the hrct of patients with Covid 19

Method: 92 patients confirmed by RT_PCR in Razi Hospital in Rasht were included in the study. In addition to their demographic and clinical information, their hrct findings were collected for evaluation. Among 92 patients (47 men, 45 women), the earliest symptoms included cough (81.5\%), fever (67.4\%), and muscle pain (58.7\%) And Six patients required intubation and mechanical ventilation at some point during their hospital stay.

Results: The most common hrct findings in our study were grade 4, peripheral lesion $87 \%$, bilateral $95.7 \%$, mixed $76.08 \%$, ggos $75 \%$, left lower lobe $94.56 \%$. Among 92 patients, we had some inconsistent findings including two patients with cardiomegaly, three patients with pleural effusion, and bilateral pleural effusion in only one patient

Conclusion: Compared to other studies, it can be concluded that peripheral, bilateral, mixed, ggos, left lower lobe involvement are important findings in most patients with covid 19. We suspect that the disease has more severe lung involvement in men and less involvement in women, which needs further investigation.

\section{Background}

In December 2019, a new pathogen COVID-19 was identified in Wuhan, Hubei Province, China that caused pneumonia and death [1, 2]. COVID-19 is one of the positive-strand RNA viruses that genetically are similar to the acute respiratory syndrome virus (SARS-COV) and the Middle East respiratory syndrome (MERS-COV) [1, 3, 4]. Following the spread of the virus to other countries such as South Korea, Italy, Iran, and Japan and reporting a death in infected people there the World Health Organization (WHO) declared it as a pandemic and named it COVID-19[3,5]. Until the date of writing this scientific research, according to WHO, 35,027,546 cases of COVID-19 have been confirmed, including 1,034,837 death[6]. COVID-19 and SARS have many close clinical characters [3]. The spread and contagion of the virus happen through large droplets created during sneezing and coughing of asymptomatic and asymptomatic patients[7]. The virus can survive on different surfaces such as metal, glass, or plastic for up to 9 days and infect people by touching the nose, eyes, and mouth $[5,7]$. After contact with the virus, symptoms may appear within 2 to 14 days [8]. The transmission rate of SARS-CoV-2 is high between families and friends who had close contact with patients or asymptomatic carriers even with a low infective dose [9].

Elderly persons with comorbidities or underlying diseases such as diabetes, hypertension, or cardiovascular diseases (CVD), are at serious and greater risk because these diseases may weaken the immune system $[3,9,10]$. Although everyone is at risk, the average age of patients was between 47-59 years old, of which women make up $41.9-45.7 \%$ of patients[7, 9], COVID-19 has various clinical features, from an asymptomatic state to acute respiratory distress syndrome until multi-organ dysfunction[7]. 
Common clinical symptoms of COVID-19 fever, nonproductive cough, dyspnea with or without diarrhea, myalgia, fatigue, usually normal or reduced leukocyte amounts, and radiographic data of pneumonia, which are similar to the symptoms of SARS-CoV and MERS-CoV contagions $[1,4,7]$. The most and main common symptom for screening is fever [3]. Pneumonia and kidney failure may happen in severe cases which lead to death [10]. In the second week, the infection progresses to hypoxemia, respiratory problems, and acute respiratory distress syndrome (ARDS), which leads to the need for mechanical ventilation in the intensive care unit (ICU) with isolated services[1]. Patients should drink plenty of fluids and electrolytes and appropriate oxygen therapy or treatment should be provided through the oxygen mask, nasal cannula, or high flow nasal oxygen therapy. In this situation, checking some parameters like heart rate, blood pressure, pulse oxygen saturation, and respiratory rate is essential[10].

COVID-19 diagnostic tests are varied and include nucleic acid detection, CT scan, immune identification technology, Real-Time-PCR, ELISA, blood culture $[4,10]$. CT imaging normally appearances infiltrate ground-glass opacities (GGOs), and subsegmental consolidation [7] single or numerous agglomerated or scattered patchy GGOs segregated by grid-like condensed or honeycomb-like interlobular septa and pulmonary consolidation with air bronchogram. The results may differ according to disease phase, patient age, and immune status at the time of imaging [10]. For identification, High-Resolution Computed Tomography (HRCT) is more sensitive than chest $x$-ray[11] and this is necessary for quick diagnosis and assessment of COVID-19 patient's severity, discovering lung deformities, clinical classification, finding of pulmonary problems, and follow-up after discharge[4, 12].

\section{Patients And Methods}

\section{Study design and patients}

This retrospective cross-sectional study was conducted from December 2019 to March 2020 on 92 cases of COVID-19 confirmed by RT-PCR in Razi Hospital, Rasht, Iran. The study was performed in accordance with the declaration of Helsinki and approved by the Ethics Committee of Guilan University of Medical Sciences (IR.GUMS.REC.1398.543). written informed consent to participate in the study was obtained from participants. Their demographic and clinical data including age, sex, underlying diseases, signs and symptoms, length of hospital stay, hospital outcome (mechanical oxygen demand (intubation) - blood oxygen saturation, recovery or death, and HRCT radiographic results were recorded and analyzed. The criterion for patient admission was the presence of a definite laboratory result to confirm the occurrence of COVID-19 in the patient file. Patient files with incomplete information (main variables like lack of definitive result of COVID- and also lack of HRCT image in the file report, were excluded from the study.

\section{Imaging analysis and quantification}

Each case underwent chest high-resolution CT (HRCT) examination within 24 hours after admission. All imaging was taken by a General Hi-speed Dual CT scanner. Then rewired and evaluated by an experienced radiologist. The imaging procedure was performed according to the common protocol of the chest. The HRCT in axial sections was used without the contrast material with a section thickness of 2 
$\mathrm{mm}$. The low-dose protocol (low-dose computed tomography) with radiation conditions of Kvp $=100-$ $120, \mathrm{mAs}=50-100$, and pitch $=1 \mathrm{~mm}$ was used. Lesion image analysis included the number of lesions, distribution of lung lobes, location of lesions in the lung, lesion size, the density of right upper lobe lesions, right middle lobe, lower right lobe, left upper lobe and lower left lobe. The location of the lesions in the lung included peripheral (if it is located in the outer third of the lung), central (inner 2/3 region of the lung), peripheral-central, and bilateral or unilateral. Lesion size was defined as: no grade, grade $1(1 \mathrm{~cm}>)$, grade $2(1<X<3 \mathrm{~cm})$, grade 3 ( $3 \mathrm{~cm}$ to $<50 \%$ of the lobe), and grade 4 ( $50-100 \%$ of lobes). The presence of ground-glass opacities, consolidation, mixed, fibrous stripes, reticulation or interstitial thickness, air bronchogram in each patient's chest CT image were assessed as lesions density. pleural effusion and cardiomegaly were also present. The patient puts his arm above his head and lies on the CT scanner. CT scanning started from the apex to the lowest part of the lungs in deep inspiration. The patient was told to hold his breath during the imaging.

\section{Statistical analysis}

In this study, the collected data is recorded in an Excel program and then coded and entered into SPSS software. Qualitative and Quantitative variables (mean and standard deviation) have been described using numbers and percentages. The Kolmogorov test was used to determine the normality of the variables. If normal, parametric tests and otherwise according to the variables, qualitative research using the chi-square test and Spearman correlation coefficient can be used to determine the frequency and correlation. Patients were divided into 4 groups for more analysis according to age as follows: group $1,<$ 20 years old; group 2, 21-40 years old; group 3, 41-60 years old; group 4, 61-80 years old and group 5, 81-100 years old.

\section{Results}

In the present study, 92 patients with PCR-confirmed COVID-19 were evaluated for HRCT in Guilan province, which is one of the COVID-19 referral centers in Iran. They were 47 men, 45 women with a mean age of $56.52 \pm 14.05$. Mean hospital stay time was $7.2 \pm 9.93$ days. Among these patients, 90 patients $(8.97 \%)$ were referred to the hospital in person. Of all patients, 75 cases had not been in direct or indirect contact with other COVID-19 patients. The baseline data are recorded in (Table 1). 
Table 1

Baseline Characteristics in COVID-19 patients.

\begin{tabular}{|ll|}
\hline Gender & M:47 (51.1\%), \\
& F:45 (48.9\%) \\
\hline REFER & $2(2.2 \%)$ \\
EMS & $90(97.8)$ \\
PERSONAL & \\
DEATH & $11(12 \%)$ \\
AGE & $56.52 \pm 14.05$ \\
\hline HOSPITALIZATION & $7.2 \pm 9.93$ \\
\hline
\end{tabular}

At first presentation, the most common early symptoms in the patients with COVID-19 in the present study, were cough ( 75 cases), fever ( 62 cases), muscle pain (54 cases), respiratory distress (34 cases), and other cases are shown in Table 2. These clinical manifestations in infected patients of different age groups were not statistically significant. After Antiviral treatment, 81 patients were discharged from the hospital but 11 patients died during hospitalizing. 30 of 92 patients had a history of at least one underlying disease (Table 4), which diabetes was the most common ( 10 cases). $O_{2}$ pressure in 25 cases was more than $93 \mathrm{~mm} \mathrm{Hg}$ and in 67 cases was less than $93 \mathrm{~mm} \mathrm{Hg}$. Six patients required intubation and mechanical ventilation at some point during their hospital stay and three of them died.

Two patients were required to undergo dialysis (Table 3). We decided to study patients separately by gender and age for a more detailed analysis. 
Table 2

Clinical information of 92 patients infected with SARS-CoV-2

\begin{tabular}{|lll|}
\hline & YES & NO \\
\hline CONTACT HISTORY & $17(18.5 \%)$ & $75(81.5 \%)$ \\
\hline FEVER & $62(67.4 \%)$ & $30(32.6 \%)$ \\
\hline COUGH & $75(81.5 \%)$ & $17(18.5 \%)$ \\
\hline MUSCLE PAIN & $54(58.7 \%$ & $38(41.3 \%)$ \\
\hline DYSTRESS & $34(37 \%)$ & $58(63 \%)$ \\
\hline CONSCIOUSNES & $2(2.2 \%)$ & $90(97.8 \%)$ \\
\hline OLFACTORY & $7(7.6 \%)$ & $85(92.4 \%)$ \\
\hline TASTE & $5(5.4 \%)$ & $87(94.6 \%)$ \\
\hline CONVULSIONS & 0 & $92(100 \%)$ \\
\hline HEADACHE & 0 & $92(100 \%)$ \\
\hline VERTIGO & 0 & $92(100 \%)$ \\
\hline PARALYSIS & 0 & $92(100 \%)$ \\
\hline PLAGUE & 0 & $92(100 \%)$ \\
\hline CHES PAIN & 0 & $92(100 \%)$ \\
\hline SKIN & 0 & $92(100 \%)$ \\
\hline STOMACHACHE & 0 & $92(100 \%)$ \\
\hline NAUSEA & 0 & $92(100 \%)$ \\
\hline VOMITING & 0 & $92(100 \%)$ \\
\hline DIARRHEA & 0 & $92(100 \%)$ \\
\hline ANOREXIA & $1(1.1 \%)$ & $91(98.9 \%)$ \\
\hline SMOOKING & $2(2.2 \%)$ & $90(97.8 \%)$ \\
\hline DRUG & 00 & $92(100 \%)$ \\
\hline
\end{tabular}


Table 3

Special actions taken

\begin{tabular}{|lll|}
\hline & YES & NO \\
\hline INTUBATION & $6(6.5 \%)$ & $86(93.5 \%)$ \\
\hline O2 & $25(27.2 \%)>93$ & $67(72.8 \%)<93$ \\
\hline DIALYSIS & $2(2.2 \%)$ & $90(97.8 \%)$ \\
\hline
\end{tabular}

Table 4

Infected patients underlings diseases information

\begin{tabular}{|lll|}
\hline & YES & NO \\
\hline CANCER & $2(2.2 \%)$ & $90(97.8 \%)$ \\
\hline LIVER & 0 & $92(100 \%)$ \\
\hline DIABET & $10(10.9 \%)$ & $82(89.1 \%)$ \\
\hline HEMATOLOGY & $2(2.2 \%)$ & $90(97.8 \%)$ \\
\hline AIDS & 0 & $92(100 \%)$ \\
\hline IMMUNIDEFICIENCY & $3(3.3 \%)$ & $89(96.7 \%)$ \\
\hline HEART & $8(8.7 \%)$ & $84(91.3 \%)$ \\
\hline KIDNEY & $6(6.5 \%)$ & $86(93.5 \%)$ \\
\hline PREGNANCY & 0 & $92(100 \%)$ \\
\hline ASTHMA & $3(3.3 \%)$ & $89(96.7 \%)$ \\
\hline OTHER LUNG disease & $2(2.2 \%)$ & $90(97.8 \%)$ \\
\hline NERVE & $2(2.2 \%)$ & $90(97.8 \%)$ \\
\hline ANOTHER chronic disease & $7(7.6 \%)$ & $85(92.39 \%)$ \\
\hline Hypertension & $7(7.6 \%)$ & $85(92.39 \%)$ \\
\hline
\end{tabular}

The present study showed that HRCT image lesions size in all patients, grade 4 (50-100 \% of the lobe) were the most common. As shown in Table 5, The most common grade was 4 (seventeen cases) in men, 1 and 2 grades in women (twelve cases each). In the age groups of 20-40, grade 2 and $60-80$, grade 3, in 80 to 100 , grade 3 were detected in HRCT. 
Table 5

A comparison of the grade of lung lesions at different ages and gender.

\begin{tabular}{|c|c|c|c|c|c|c|c|c|c|}
\hline & & & & \multicolumn{5}{|c|}{ grade } & \multirow[t]{2}{*}{ Total } \\
\hline & & & & .00 & 1.00 & 2.00 & 3.00 & 4.00 & \\
\hline \multirow[t]{4}{*}{ gender } & \multirow[t]{2}{*}{$\mathrm{F}$} & \multicolumn{2}{|l|}{ Count } & 0 & 13 & 14 & 6 & 12 & 45 \\
\hline & & \multicolumn{2}{|c|}{$\%$ within gender } & $0.0 \%$ & $28.8 \%$ & $31.1 \%$ & $13.3 \%$ & $26.6 \%$ & $100.0 \%$ \\
\hline & $M$ & \multicolumn{2}{|l|}{ Count } & 0 & 10 & 11 & 9 & 17 & 47 \\
\hline & & \multicolumn{2}{|c|}{$\%$ within gender } & $0.0 \%$ & $21.2 \%$ & $21.4 \%$ & $19.4 \%$ & $36.1 \%$ & $100.0 \%$ \\
\hline \multirow[t]{2}{*}{ Total } & \multicolumn{3}{|c|}{ Count } & 0 & 23 & 25 & 15 & 29 & 92 \\
\hline & \multicolumn{3}{|c|}{$\%$ within gender } & $0.0 \%$ & $25.0 \%$ & $27.1 \%$ & $16.3 \%$ & $31.5 \%$ & $100.0 \%$ \\
\hline \multirow[t]{2}{*}{$21-40$} & \multicolumn{2}{|c|}{ Count } & 0 & & 5 & 7 & 2 & 3 & 17 \\
\hline & \multicolumn{2}{|c|}{$\%$ within age } & $0.0 \%$ & & $29.4 \%$ & $41.1 \%$ & $11.7 \%$ & $17.6 \%$ & $100.0 \%$ \\
\hline \multirow[t]{2}{*}{$41-60$} & \multicolumn{2}{|c|}{ Count } & 0 & & 11 & 10 & 4 & 13 & 38 \\
\hline & \multicolumn{2}{|c|}{$\%$ within age } & $0.0 \%$ & & $28.9 \%$ & $26.3 \%$ & $10.5 \%$ & $34.2 \%$ & $100.0 \%$ \\
\hline \multirow[t]{2}{*}{$61-80$} & \multicolumn{2}{|c|}{ Count } & 0 & & 6 & 7 & 7 & 12 & 32 \\
\hline & \multicolumn{2}{|c|}{$\%$ within age } & $0.0 \%$ & & $18.7 \%$ & $21.8 \%$ & $21.8 \%$ & $37.5 \%$ & $100.0 \%$ \\
\hline \multirow[t]{2}{*}{$81-100$} & \multicolumn{2}{|c|}{ Count } & 0 & & 1 & 1 & 2 & 1 & 5 \\
\hline & \multicolumn{2}{|c|}{$\%$ within age } & $0.0 \%$ & & $20.0 \%$ & $20.0 \%$ & $40.0 \%$ & $20.0 \%$ & $100.0 \%$ \\
\hline \multirow[t]{2}{*}{ Total } & \multicolumn{2}{|c|}{ Count } & 0 & & 23 & 25 & 15 & 29 & 92 \\
\hline & \multicolumn{2}{|c|}{$\%$ within age } & $0.0 \%$ & & $25.0 \%$ & $27.17 \%$ & $16.3 \%$ & $31.5 \%$ & $100.0 \%$ \\
\hline
\end{tabular}

We found that lesions were more localized in the peripheral zone (80 cases_87\%). The involvement of peripheral lung lesions in the age of 40-60 was more than in other age groups. Also, lesions were involved in the peripheral-central zones in twelve cases (13\%).

Based on the HRCT finding, 72 patients (78.3\%) had lesions in all lung lobes. Our result showed that the most common site of involvement was the left lower lobe (87 cases) at different ages and the least involved lobe was the upper left (81 cases). The most common site of involvement lobes in men was almost all of them, but in women lower left was most common. We also diagnosed 88 patients (95.7\%) with bilateral lung involvement, three patients (3.3\%) with only the left lung, and one patient $(1.1 \%)$ with only the right lung.

We also analyzed the characteristics of each patient lesion. 297 lesions in HRCT calculated for all infected individuals, in total, mixed (70 cases) and GGOs (69 cases) are a commonly observed HRCT 
finding in lung lesions in patients. In men, the most common lesion was mixed, but in women, GGOs. (Table 6)

Table 6

Presented findings in HRCT scan of symptomatic patients infected with coronavirus 2019

\begin{tabular}{|lll|}
\hline PERIPHERAL & $\mathbf{8 0}$ & $\mathbf{8 7 \%}$ \\
\hline PERIPHERAL-CENTRAL & 12 & $13 \%$ \\
\hline Bilateral & 88 & $95.7 \%$ \\
\hline left & 3 & $3.3 \%$ \\
\hline right & 1 & $1.1 \%$ \\
\hline mixed & 70 & $76.08 \%$ of cases \\
\hline Ground glass op & 69 & $75.00 \%$ \\
\hline Consolidation & 52 & $56.52 \%$ \\
\hline Reticulation or interstitial thickening & 50 & $54.34 \%$ \\
\hline Fibrous strips & 40 & $43.47 \%$ \\
\hline Air bronchogram & 16 & $17.39 \%$ \\
\hline Lower left & 87 & $94.56 \%$ of cases \\
\hline Lower right & 86 & $93.47 \%$ \\
\hline Upper right & 86 & $93.47 \%$ \\
\hline Middle right & 83 & $90.21 \%$ \\
\hline Upper left & 81 & $88.04 \%$ \\
\hline
\end{tabular}

For each patient lesion, an air bronchogram was evaluated, which was a total of 16 . There was no significant difference between the different age groups, but it was seen more in men (11 cases) than women (5 cases).

Among 92 patients, we had some inconsistent findings including two patients with cardiomegaly, three patients with pleural effusion, and bilateral pleural effusion in only one patient

\section{Discussion}

The SARS-COV-2 disease is considered a global threat with a high infectious rate and CT imaging has an important role in its rapid diagnosis and evaluation $[13,14]$. To our knowledge, this is the first study about hospitalized patients from Guilan province by HRCT for diagnosis and evaluation. The present study was performed HRCT on 92 patients and recorded signs of involvement in the CT images. In the present study, 
the cough was found to be the most common clinical manifestation (81.52\%). This rate in another study with 120 patients was $90 \%$ [8] (5) which is close to our rate. However, in other studies fever was the most common symptom $[10,14,15]$. In another similar study in Iran, dyspnea was the most common symptom in patients [16], Our results showed that $32.60 \%$ of patients had a history of an underlying disease which this rate was $18.48 \%[16]$ and 34.9\%[10] in other studies. In contrast, /diabetic disease was the most contributor to disease in the present study, but study [16] declared cardiovascular disease was the most. Six out of 92 patients $(6.52 \%)$ required mechanical ventilation, in contrast, whit other study results which this rate was $13(10.83 \%)[8]$.

The most common presented findings in patients, peripheral distribution, and lower left lobe involvement, grad 4 of lesion sizes were mixed. The prevalence of mixed in the studied population was $76.08 \%$, which is systematic and meta-analysis study was $43.0 \%$ [17]. Also in this study, the prevalence of ground-glass opacity was $75 \%$, which varied from $64.6-100 \%$ in other studies $[8,17]$ In a similar study in Iran [13], GGOs were the most symptom in CT images (87.3\%). The peripheral zone was the second most common finding in this study, observed in $87 \%$ of the study population. this rate was reported in other studies $57.1 \%[17](6)$ and $82.4 \%[13]$. Our results showed the most site of the involved lobe was the left lower lobe in $94.56 \%$ of the population. In similar systematic and meta-analyses [17], LLL was the most common $(71.2 \%)$. In another study, the lesions of patients were mainly located in the lower lobe of the right lung [15].

\section{Conclusion}

Compared to other studies, it can be concluded that peripheral, bilateral, mixed, ggos, left lower lobe involvement are important findings in most patients with covid 19. We suspect that the disease has more severe lung involvement in men and less involvement in women, which needs further investigation.

\section{Declarations}

\section{Acknowledgments}

We are especially grateful to all the experts who were an integral partners in the preparation of facilities.

\section{Authors' contributions}

C E-A design the study, interpretation of data, reading; $Z \mathrm{~J}$ : design the study, reading and approving; M A K $\mathrm{G}$ collecting data; $\mathrm{H} \mathrm{H}$ interpretation of data; T Y K data collection and data sorting; AA F collecting data; S R collecting data; AS-N data sorting; H S interpretation of data; B G-M writing of the article and revision its content; $\mathrm{H} \mathrm{K}$ and M A revision its content; S M M and H S E-S and Z G G collecting data ; $\mathrm{S}$ contributed to image diagnosis, provided critical reviewand substantially revised the manuscript;M F provided critical reviewand substantially revised the manuscript .All authors read and approved thefinal manuscript. 


\section{Funding}

This study was supported financially by the research council from Guilan University of Medical Sciences [IR.GUMS.REC.1398.543]

\section{Availability of data and materials}

Availability of data and materials The datasets generated and/or analyzed in the current study are not publicly available due to patient privacy protection but are available from the corresponding author on reasonable request.

\section{Ethics approval and consent to participate}

The study was performed in accordance with the declaration of Helsinki and approved by the Ethics Committee of Guilan University of Medical Sciences (IR.GUMS.REC.1398.543).

\section{Consent for publication}

written informed consent to participate in the study was obtained from participants.

\section{Competing of interest}

The authors declare that they have no conflict of interest concerning this article.

\section{Author details}

${ }^{1}$ Department of Anesthesiology, School of Medicine Neuroscience Research Center, Poursina Hospital, GuilanUniversity of Medical Sciences ${ }^{2}$ Razi Clinical Research Development Unit, Guilan University of Medical Sciences, Rasht, Iran ${ }^{3} \mathrm{MSc}$ in Critical Care Nursing, Guilan University of Medical Sciences, Rasht, Iran ${ }^{4}$ Department of Vascular Surgery, Guilan University of Medical Sciences, Rasht, Iran ${ }^{5}$ Assistant Professor of Infectious Disease Department of Health, Nutrition \& Infectious Diseases, School of Medicine Razi Hospital Guilan University of Medical Sciences ${ }^{6}$ Inflammatory Lung Diseases Research Center, Department of Internal Medicine, Razi Hospital, School of Medicine, Guilan University of Medical Sciences, Rasht, Iran ${ }^{7}$ Department of Anesthesiology, School of Medicine Velayat Hospital Guilan University of Medical Sciences ${ }^{8}$ Department of Anesthesiology, School of Medicine Heshmat Hospital Guilan University of Medical Sciences ${ }^{9}$ Department of physics, Faculty of Science, University of Guilan, Rasht, Iran 
${ }^{10}$ Department of Radiology, School of Medicine Poursina Hospital Guilan University of Medical Sciences ${ }^{11}$ Department of Physiology, Faculty of Veterinary Medicine, Science and Research Branch, Islamic Azad

12Department of Anesthesiology.Anesthesia research center, Guilan University of Medical Sciences, Rasht, Iran

\section{References}

1. Kannan S, Shaik Syed Ali P, Sheeza A, Hemalatha K. COVID-19 (Novel Coronavirus 2019) - recent trends. European review for medical and pharmacological sciences. 2020;24(4):2006-11.

2. Sun J, He WT, Wang L, Lai A, Ji X, Zhai X, et al. COVID-19: Epidemiology, Evolution, and CrossDisciplinary Perspectives. Trends in molecular medicine. 2020;26(5):483-95.

3. Nikolich-Zugich J, Knox KS, Rios CT, Natt B, Bhattacharya D, Fain MJ. SARS-CoV-2 and COVID-19 in older adults: what we may expect regarding pathogenesis, immune responses, and outcomes. GeroScience. 2020;42(2):505-14.

4. Li X, Geng M, Peng Y, Meng L, Lu S. Molecular immune pathogenesis and diagnosis of COVID-19. Journal of pharmaceutical analysis. 2020;10(2):102-8.

5. Pascarella G, Strumia A, Piliego C, Bruno F, Del Buono R, Costa F, et al. COVID-19 diagnosis and management: a comprehensive review. Journal of internal medicine. 2020;288(2):192-206.

6. Sachs JD, Horton R, Bagenal J, Amor YB, Caman OK, Lafortune G. The Lancet COVID-19 Commission. The Lancet. 2020;396(10249):454-5.

7. Singhal T. A Review of Coronavirus Disease-2019 (COVID-19). Indian journal of pediatrics. 2020;87(4):281-6.

8. Esakandari H, Nabi-Afjadi M, Fakkari-Afjadi J, Farahmandian N, Miresmaeili SM, Bahreini E. A comprehensive review of COVID-19 characteristics. Biological procedures online. 2020;22:19.

9. Guo YR, Cao QD, Hong ZS, Tan YY, Chen SD, Jin HJ, et al. The origin, transmission and clinical therapies on coronavirus disease 2019 (COVID-19) outbreak - an update on the status. Military Medical Research. 2020;7(1):11.

10. Chakraborty C, Sharma AR, Sharma G, Bhattacharya M, Lee SS. SARS-CoV-2 causing pneumoniaassociated respiratory disorder (COVID-19): diagnostic and proposed therapeutic options. European review for medical and pharmacological sciences. 2020;24(7):4016-26.

11. Raghuvanshi V, Sood RG, Jhobta A, Sarkar M, Tomar A, Khanna S. Use of High-Resolution Computed Tomography (HRCT) in Diagnosis of Sputum Negative Pulmonary Tuberculosis. Turkish thoracic journal. 2016;17(2):59-64.

12. Gao L, Zhang J. Pulmonary High-Resolution Computed Tomography (HRCT) Findings of Patients with Early-Stage Coronavirus Disease 2019 (COVID-19) in Hangzhou, China. Medical science monitor : international medical journal of experimental and clinical research. 2020;26:e923885. 
13. Majidi H, Bani-Mostafavi ES, Mardanshahi Z, Godazandeh F, Ghasemian R, Heydari K, et al. Highresolution computed tomography finding in 552 patients with symptomatic COVID-19: first report from north of Iran. Emergency radiology. 2020;27(6):633-9.

14. Dai H, Zhang X, Xia J, Zhang T, Shang Y, Huang R, et al. High-resolution Chest CT Features and Clinical Characteristics of Patients Infected with COVID-19 in Jiangsu, China. International journal of infectious diseases : IJID : official publication of the International Society for Infectious Diseases. 2020;95:106-12.

15. Chen Z, Fan H, Cai J, Li Y, Wu B, Hou Y, et al. High-resolution computed tomography manifestations of COVID-19 infections in patients of different ages. European journal of radiology. 2020;126:108972.

16. Zamanian M, Foroozanfar Z, Izadi Z, Jafari S, Derakhshankhah H, Salimi M, et al. Association of Underlying Diseases and Clinical Characteristics with Mortality in Patients with 2019 Novel Coronavirus in Iran. Arch Clin Infect Dis. 2020;15(5):e104621.

17. Muhammad SZ, Ahmed A, Shahid I, Khalid A, Menezes RG, Sheikh MU, et al. Chest computed tomography findings in hospitalized COVID-19 patients: a systematic review and meta-analysis. Le infezioni in medicina. 2020;28(3):295-301. 